\title{
DISPOSICIÓN DEL DERECHO A LA VIDA DE LOS MENORES DE EDAD: UNA NECESARIA DISCUSIÓN SOBRE EUTANASIA Y SUICIDIO ASISTIDO EN CHILE
}

\author{
Estefanía Esparza Reyes ${ }^{1}$
}

Resumen: Tanto la eutanasia como el suicidio asistido no han sido temas suficientemente explorados por la doctrina chilena, falta de tratamiento que se agudiza al referirse a los menores de edad. El presente artículo tiene por objetivo dejar en evidencia que las principales disposiciones de derechos fundamentales relacionadas con la eutanasia y el suicidio asistido en menores de edad —el derecho a la vida y los derechos de los niños, niñas y adolescentes - admiten diversas interpretaciones y, en consecuencia, pueden ser utilizadas para argumentar a favor y en contra de la legalización de dichos fenómenos. Tras una breve definición de los conceptos a los que se hace alusión y un panorama general de la regulación comparada, se abordan los principales derechos involucrados, dejando en evidencia que los supuestos impedimentos jurídicos para la despenalización de la eutanasia y suicidio asistido son interpretaciones de los derechos involucrados, las cuales no resultan del todo concordantes con las actuales tendencias de la teoría de los derechos fundamentales y que, por ello, la sola variación en esta interpretación permitiría abrir la posibilidad de una regulación que, al menos, no sancione en todos los casos la eutanasia y/o el suicidio asistido de los menores de edad en Chile.

Palabras clave: eutanasia; suicidio asistido; vida; menores de edad; derechos humanos

Legal provision for the right of life of minors: a necessary discussion about euthanasia and assisted suicide in Chile

Abstract: Both euthanasia and assisted suicide have not been sufficiently explored by Chilean experts, lack of reflection heightened when referring to minors. This article aims to make clear that the main provisions of fundamental rights related to euthanasia and assisted suicide in minors, that is the right to life and the rights of children and adolescents, admit diverse interpretations and, therefore, they may be used to argue in favor or against the legalization of such issues. After a brief definition of the concepts considered and a general view of compared legislation, the main rights involved are addressed, making clear that the supposed legal impediments to decriminalization of euthanasia and assisted suicide, are interpretations of rights involved, which they are not completely concordant with current tendencies about the theory of fundamental rights and, therefore, the only variation in interpretation will allow to open the possibility of a regulation that, at least, will not penalize all cases of euthanasia and assisted suicide of minors in Chile.

Keywords: euthanasia, assisted suicide, life, minors, human rights

Normas do direito à vida dos menores de idade: uma discussáo necessária sobre eutanásia e suicídio assistido no Chile

Resumo: Tanto a eutanásia como o suicídio assistido não tem sido temas suficientemente explorados pela doutrina chilena, falta de tratamento que se agudiza ao referir-se aos menores de idade. O presente artigo tem por objetivo deixar em evidência que as principais normas de direitos fundamentais relacionadas com a eutanásia e o suicídio assistido em menores de idade - o direito à vida e os direitos dos meninos, meninas e adolescentes - admitem diversas interpretaçóes e, em consequência, podem ser utilizadas para argumentar a favor ou contra a legalização de tais fenômenos. Depois de uma breve definição dos conceitos a se faz alusão e um panorama geral da regulação comparada, abordam-se os principais direitos envolvidos, deixando em evidência que os supostos impedimentos jurídicos para a descriminalização da eutanásia e do suicídio assistido, são interpretaçóes dos direitos envolvidos, as quais não resultam completamente concordantes com as atuais tendências da teoria dos direitos fundamentais y que por isto, só esta variação na interpretação permitiria abrir a possibilidade de uma regulação que, ao menos, não sancione em todos os casos a eutanásia e/ou o suicídio assistido de menores de idade no Chile.

Palavras-chave: eutanásia, suicídio assistido, vida, menores de idade, direitos humanos

1 Departamento de Ciencias Jurídicas Universidad de La Frontera, Temuco, Chile

Correspondencia: estefania.esparza@ufrontera.cl 


\section{Introducción}

Como es sabido, actualmente, nuestro país no permite la eutanasia, ni activa ni pasiva (ni de doble efecto) y tampoco el suicidio o muerte asistida. Sobre estos conceptos existe enorme disparidad a la hora de definirlos.

En relación a la eutanasia, encontramos a quienes sostienen que resulta imprescindible que la persona que realiza o dirige el procedimiento sea un profesional de la salud(1), mientras que, para otros, tal atributo no sería esencial(2). Así, la calidad del sujeto que realiza la acción no ha sido el único tema de discusión, puesto que en su conceptualización también puede verificarse discrepancias en relación a las condiciones del sujeto pasivo, es decir si debe(2) o no (3) encontrarse en una situación próxima a la muerte; en las clasificaciones de la eutanasia ${ }^{3}$; o si es necesario(4) o no(5), que la muerte se produzca sin dolor; exigencia de la voluntariedad de la conducta(6) o no(7), entre muchas otras.

Algo relativamente similar ocurre con el suicidio asistido, con el que tampoco existe acuerdo sobre la necesidad de intervención de profesionales del área médica o, en su defecto, bastaría con el auxilio que cualquier persona pueda entregar a quien pretende terminar con su vida(6).

Para estos efectos entenderemos que "eutanasia es toda conducta de un médico, u otro profesional sanitario bajo su dirección, que causa de forma directa la muerte de una persona que padece una enfermedad o lesión incurable con los conocimientos médicos actuales que, por su naturale$\mathrm{za}$, le provoca un padecimiento insoportable y le causará la muerte en poco tiempo. Esta conducta responde a una petición expresada de forma libre y reiterada, y se lleva a cabo con la intención de liberarle de este padecimiento, procurándole un bien y respetando su voluntad acciones (u omis-

${ }^{2}$ Se trataría, además, de la postura de la Iglesia católica, debido a la definición de eutanasia que realiza la Declaración "Iura et Bona", Sagrada Congregación para la doctrina de la Fe. Declaración "Iura Et Bona" sobre la Eutanasia 1980. Disponible en http://www.vatican. va/roman_curia/congregations/cfaith/documents/rc_con_cfaith_ doc_19800505_euthanasia_sp.html

${ }^{3}$ Solo a modo de ejemplo, se ha distinguido entre eutanasia activa, pasiva o de doble efecto; natural o provocada; autónoma o heterónoma; directa o indirecta; piadosa, eugenésica o económica; distanasia; adistanasia y ortotanasia, entre muchos otros. iones) realizadas deliberadamente por un médico, u otro profesional sanitario bajo su dirección, que tienen como objetivo provocar directamente la muerte a petición expresa, voluntaria y reiterada de un paciente capaz que presenta un sufrimiento intenso, físico o psíquico, a consecuencia de una enfermedad terminal o irreversible y que el propio paciente experimenta como algo inaceptable, insoportable e indigno" $(8,1)$. De igual manera, se considerará suicidio o muerte asistida la muerte voluntaria que se realiza con la colaboración de un tercero por petición del sujeto pasivo.

Tanto la eutanasia cuanto el suicidio asistido han despertado un enorme interés, pues se trata de una de las materias en las cuales se reflejan nuestras convicciones personales, éticas y religiosas, y que expresan la diversidad de los seres humanos. Sin embargo, el tema se complejiza todavía más si nos enfrentamos a la posibilidad de que sean los menores de edad, niños o adolescentes, quienes puedan solicitar y obtener autorización para la realización de esta clase de conductas. De esta forma, es posible sostener que si bien, la eutanasia y el suicidio asistido han sido abordados por los autores, no ha existido tal preocupación en caso de ser menores de edad quienes pretendan realizar dicha conducta.

La presente reflexión tiene especial importancia en el caso de los adolescentes, es decir, personas entre los 10 y los 19 años(9), pero todavía más precisamente entre los 10 y los 18 ańos, debido a que en la mayoría de los países se posee plena capacidad jurídica a los 18 años, siendo también concordante con los establecido en la Convención Internacional sobre los Derechos del Niño ${ }^{4}$.

\section{Breve panorama general}

\section{La actualidad internacional}

En otras latitudes, la posibilidad de permitir la eutanasia o el suicidio asistido a menores de edad presenta diversos grados de desarrollo. Así, mientras nos encontramos con países que no lo admiten en mayores ni menores de edad (Espańa), otros países penalizan la eutanasia, pero autori-

\footnotetext{
${ }^{4}$ Artículo 1 CIDN: "Para los efectos de la presente Convención, se entiende por nińo todo ser humano menor de dieciocho ańos de edad, salvo que, en virtud de la ley que le sea aplicable, haya alcanzado antes la mayoría de edad".
} 
zan algunos casos de suicidio asistido (Alemania); permiten eutanasia y suicidio asistido solo en mayores de edad (Luxemburgo), otros recogen solo la eutanasia en mayores y menores de edad, no accediendo al suicidio asistido (Bélgica); naciones que permiten solo la eutanasia en personas mayores de 12 años (Colombia); países que permiten solo el suicidio asistido en mayores de edad, proscribiendo la eutanasia (Suiza), y, por último, países que admiten eutanasia y suicidio asistido en mayores y menores de edad (Holanda).

Con todo, es el caso holandés el que resulta más llamativo, pues mediante la exclusión de estas conductas del delito de homicidio (art. 293 del Código Penal) y del de auxilio al suicidio (art. 294.2. del Código Penal), permite la eutanasia y el suicidio asistido si se cumplen ciertos supuestos de hecho, especialmente referidos a información, voluntariedad, sufrimiento y falta de mejoría.

Por otra parte, la ley distingue entre mayores y menores de edad y, en este último caso, podemos encontrar distintas categorías y requisitos para obtener la eutanasia o el suicidio asistido.

a) Menores de 12 años: no se hace referencia a ellos y, en consecuencia, aplicando las reglas generales, no podrían solicitar eutanasia ni suicidio asistido.

b) Adolescentes entre 12 y 16 años: dos requisitos: que estén en condiciones de realizar una valoración razonable de sus intereses en este asunto y autorización de los padres, o del padre o madre que ejerza la patria potestad o la persona que tenga su tutela.

c) Adolescentes entre 16 y 18 años: aquí nuevamente se necesario realizar una distinción:

Adolescentes que no estén en condiciones de expresar su voluntad: dos requisitos: haberse encontrado en condiciones de realizar una valoración razonable de sus intereses antes de pasar al estado actual y haber redactado una declaración por escrito que contenga la petición de terminación de su vida.

Adolescentes que están en condiciones de expresar su voluntad: dos requisitos: que estén en con- diciones de realizar una valoración razonable de sus intereses en este asunto y participación de los padres, o del padre o madre que ejerza la patria potestad o la persona que tenga su tutela, de la decisión.

Como puede notarse, los requisitos son menores en el caso de adolescentes sobre 16 años, solo requiriéndose que los padres participen de la decisión, más no que estén de acuerdo. Por otra parte, destaca que en caso de no poder expresar su voluntad, el adolescente no requiere de la autorización o participación de sus padres o tutores.

\section{La actualidad nacional}

Actualmente en Chile las figuras de eutanasia y suicidio asistido no se encuentran tipificadas como delitos de manera expresa, sino que tales conductas son constitutivas de distintos delitos penales. Así, desde una primera aproximación, podrían encontrarse incluidas en las figuras de homicidio, sea simple o calificado, parricidio, infanticidio y auxilio al suicidio. Aunque parece algo obvio, resulta relevante, en este punto, dejar en evidencia que tales delitos contemplan la protección del bien jurídico "vida", teniendo en este sentido similitudes importantes con el derecho constitucional a la vida.

Por otra parte, la Ley 20.584, que regula los derechos y deberes de las personas en relación con acciones vinculadas a su atención en salud, de 24 de abril de 2012, establece en su artículo 14 que las personas poseen el derecho a otorgar o denegar su voluntad para someterse a cualquier procedimiento o tratamiento, aunque el rechazo en ningún caso "puede tener como objetivo la aceleración artificial de la muerte, la realización de practicas eutanásicas o el auxilio al suicidio”. De igual manera, el artículo 16 de la misma ley, reforzando esta idea, señala que en el caso de personas en estado terminal y debidamente informadas, a quienes les asiste el derecho a negarse a la aplicación de un tratamiento para alargar artificialmente su vida, tal decisión no "podrá implicar como objetivo la aceleración artificial del proceso de muerte". Tal ley no contempla disposiciones especiales en relación a los menores de edad, aunque en ocasiones hace alusión a los "representantes legales", siendo estos últimos, en la práctica, quienes pres- 
tan su consentimiento en los casos que involucren niños, niñas y adolescentes.

Generalmente motivados por un caso de alta connotación pública, desde 2004 a la fecha se han presentado ocho proyectos de ley que intentan regular la eutanasia ${ }^{5}$, de los cuales dos se encuentran archivados (Boletines 3690-11 y 4201-11); uno rechazado (Boletín 9602-11) y cinco en tramitación (Boletines 7736-11; 9644-11; 11577-11; $11703-11$ y $11745-11)$. Tales proyectos definen de manera diversa la eutanasia, permitiéndola expresamente en algunos casos, mientras que en otros simplemente se excluye tal fenómeno de los delitos anteriormente señalados.

Los proyectos de ley presentados es posible dividirlos en dos grupos: el primero de ellos se caracteriza por permitir la eutanasia pasiva y activa en menores de edad sin importar la edad, correspondiendo la decisión a los padres de consuno, al que existiese a falta de alguno o, en caso de faltar ambos, a la mayoría de los parientes consanguíneos presentes de grado más próximo en la línea colateral, hasta el tercer grado inclusive (Boletines 3690-11; 4201-11 y 9602-11); mientras que el segundo grupo se caracteriza por establecer que la eutanasia solo procederá en caso de tratarse de personas mayores de edad (Boletines 7736- 11; 9644-11; 11577-11; 11703-11 y 11745-11).

Pese a lo que pueda pensarse, los primeros proyectos de ley fueron, precisamente, los que permitían la posibilidad de solicitar y obtener la eutanasia a los menores de edad, recorriendo en este punto un camino diametralmente opuesto a otras legislaciones, en las que, con el correr de los años, se ha ido permitiendo tal posibilidad.

En cualquier caso, aun existiendo voluntad política, en el sentido de autorizar la eutanasia o suicidio asistido de los menores de edad, tales iniciativas se enfrentarían a un nuevo desafío: determinar si el ordenamiento jurídico constitucional de Chile contempla tal posibilidad.

\footnotetext{
${ }^{5}$ Fecha última revisión 18 de junio de 2018. Fuente: www.camara.cl
}

La eutanasia y el suicidio asistido de los menores de edad desde una perspectiva iusfundamental. Razones jurídicas para un sí, razones jurídicas para un no

En términos muy generales, los derechos fundamentales o derechos humanos están contenidos, al menos mayoritariamente, en la Constitución y los tratados internacionales. Producto de la estructura piramidal de nuestro ordenamiento jurídico, todas las disposiciones de inferior jerarquía a la Constitución, tales como leyes, decretos y ordenanzas, deben adecuarse a $-\mathrm{O}$ al menos no contradecir - la Carta Fundamental. De esta forma, a ninguna autoridad, incluidos el legislador, el presidente de la República y los jueces, les está permitido adoptar normativas que sean contrarias a los derechos asegurados en la Constitución. En caso de incurrir en tal conducta, grosso modo, tales normas jurídicas deben ser declaradas inconstitucionales y retiradas del ordenamiento jurídico.

En el debate jurídico sobre la eutanasia y suicidio asistido es posible reconocer dos etapas diferenciadas. Con posterioridad a la Segunda Guerra Mundial se discutía solo la posibilidad de despenalizar tales conductas, mientras que actualmente existen cuestionamientos en relación a la posibilidad de que se trate de un nuevo derecho fundamental(10), el que ha sido enunciado como "derecho a morir" o "derecho a una muerte digna" ${ }^{\circ}$.

En cualquier caso, al analizar la eutanasia y el suicidio asistido en menores de edad, es posible encontrar relaciones directas y evidentes con diversos derechos fundamentales y, de manera más específica, con aspectos relevantes para la teoría general de los derechos humanos y de los valores ${ }^{7}$.

A modo meramente enunciativo, se visualizan vinculaciones con la dignidad humana, consagrada en nuestra Constitución en el artículo $1^{8}$, en

\footnotetext{
${ }^{6}$ Se ha estimado que este "derecho a morir" derivaría del derecho a la privacidad y de la dignidad humana. En otras ocasiones se ha considerado que el "derecho a morir" se encontraría comprendido en el derecho a la vida. Esta posición no ha tenido apoyo jurisprudencial y fue descartada expresamente por la Corte Europea de Derechos Humanos en múltiples ocasiones.

7 Zúñiga, por ejemplo, entiende que la eutanasia se relaciona con la dignidad humana y la libertad(11), mientras que Vivanco estima que existe una estrecha conexión con el derecho a la vida(10).

${ }^{8}$ Art. $1^{\circ}$ Constitución Política de la República de Chile: "Las perso-
} 
dos sentidos contrapuestos(12), pues cabría preguntarse si de ella se deriva una "obligación de vivir" (prohibición de la eutanasia y el suicidio asistido) o, por el contrario, el reconocimiento de la dignidad humana exigiría —o al menos autorizaría a- morir en caso de que las condiciones, como por ejemplo una enfermedad degenerativa, no se consideren acordes con tal dignidad'. En otras palabras, la dignidad ha sido utilizada para argumentar en favor y en contra de la despenalización/legalización de la eutanasia y el suicidio asistido.

Por otra parte, es posible encontrar conexiones con el valor y derecho fundamental a la libertad consagrado en los artículos 1 y 19 No 7 , en cuanto a su contenido y límites: jel derecho a la libertad incluye un derecho a morir?(13). Y, en el entendido afirmativo, ¿es factible exigir colaboración al Estado en caso de no poder ejercer directamente esta facultad?(14). Así, la libertad suele ser invocada para argumentar a favor de despenalización/ legalización de la eutanasia y el suicidio asistido.

Empero, existen dos temáticas que tradicionalmente han sido invocadas para descartar la posibilidad de una regulación que permita la eutanasia y el suicidio asistido, especialmente a los menores de edad; estas son el derecho a la vida, puesto que se ha sostenido que esta clase de conductas vulneraría tal derecho, y la titularidad y ejercicio de los derechos de los menores de edad, debido a que no existe acuerdo sobre si los menores de edad poseen y son capaces, ellos mismos, de ejercer (y renunciar) a sus derechos. A ellas se dedicarán las siguientes líneas.

\section{El derecho a la vida y la disposición de la vida}

Tal como suele ocurrir en el Derecho con un sinnúmero de temas, no existe acuerdo en doctrina sobre la calificación jurídica de la vida, es decir, si es solo un derecho o es al mismo tiempo un derecho y un deber(15). Esta crucial diferencia acarrea consecuencias disímiles a la hora de entender y aplicar el derecho a la vida, hecho que repercute

nas nacen libres e iguales en dignidad y derechos".

9 Circunstancia que ha sido reconocida por distintos tribunales constitucionales en el mundo, desde la sentencia del caso Cruzan, ocurrido en Estados Unidos de Norteamérica en 1990 (497 U.S. 261), en el cual se abordó específicamente la alimentación forzada de personas que no podían manifestar su voluntad expresamente. directamente en la posibilidad de aceptar la eutanasia y el suicidio asistido.

De esta forma, en primer término es necesario referirse a la vida entendida como derecho y deber al mismo tiempo. Pareciera ser que esta concepción de derecho y deber a la vez provendría del pensamiento de Locke y tendría importantes antecedentes en la obra de Santo Tomás de Aquino. Así, el derecho a la vida como derecho natural implicaba al mismo tiempo un deber de conservación, por cuanto se entendía que la vida pertenecía a Dios y, en consecuencia, no susceptible de disposición por parte del ser humano. Su importancia era tal que el ser humano salía del estado de naturaleza para lograr su conservación(16). Esta concepción de que algunos derechos son al mismo tiempo deberes fue adoptada y perfilada por los liberales sin mayores inconvenientes y primó hasta mediados del siglo XX.

Con el desarrollo de teorías modernas sobre los derechos fundamentales, se comenzó a cuestionar que los derechos puedan a la vez ser deberes, fenómeno que obedeció a múltiples factores. Así, de modo no taxativo, puede enunciarse la pérdida de adeptos a las teorías sobre la existencia del Derecho Natural y especialmente la dotación de una estructura de los derechos fundamentales, tarea a la que se abocó la doctrina y la jurisprudencia de diversos países. Esta estructura distingue de manera más o menos rígida entre quienes son los titulares de los derechos y los destinatarios de los mismos, estableciendo que no se puede ser a la vez titular de un derecho y obligado a respetar el mismo del cual se es titular. Esta afirmación no quiere decir que no existan obligaciones en relación a los derechos, muy por el contrario: simplemente que sobre el derecho que posee un individuo solo los terceros tienen obligaciones, son sus destinatarios. Tal aseveración debe entenderse sin perjuicio de la consagración siempre expresa de derechos que pueden ser deberes al mismo tiempo, como el derecho-deber preferente de educar a los/as hijos/as(17).

Por otra parte, la estructura que poseen los derechos fundamentales, permite sostener la imposibilidad de que uno mismo pueda vulnerar sus derechos, en este caso el derecho a la vida. Así, según la nomenclatura de Alexy, el derecho a la vida es 
un "derecho a algo"(18), una relación tríadica en la que el objeto del derecho consiste en la realización (u omisión) de una conducta por parte de un tercero. En consecuencia, como se requiere para vulnerar el derecho que se realice la conducta de un tercero, no es factible sostener que uno mismo pueda vulnerar su derecho a la vida.

De esta forma, hoy sabemos que no es posible que un derecho fundamental, como es el derecho a la vida, sea, por regla general, al mismo tiempo un deber para el titular, puesto que si bien tal derecho genera obligaciones y mandata no disponer de la vida, los destinatarios de ellas son terceros, es decir, el Estado y los particulares, mas no el propio titular, quien posee la facultad de exigir que los terceros respeten tal derecho. Así, al no existir un deber de autoconservación derivado del derecho a la vida, no sería apropiado afirmar que la eutanasia o el suicidio asistido vulneran este derecho.

En la revisión bibliográfica también es posible encontrar dos posturas contrapuestas en relación a la jerarquía del derecho a la vida dentro de los ordenamientos jurídicos. Algunos autores sostienen que el derecho a la vida es el derecho más importante de los catálogos de derechos humanos(19), postura que suele sostenerse sobre la base de que la vida sería el presupuesto básico de existencia de todos los demás derechos fundamentales; mientras que otros autores afirman que no es posible establecer una jerarquía abstracta de los derechos(18). Esta última postura resulta concordante con las actuales tendencias en materia de derechos fundamentales, pues se ha entendido que todos ellos son relevantes para la existencia humana, no pudiéndose decidir de antemano qué derecho primará en el caso concreto, pues una opción en tal sentido ocasionaría que ciertos derechos siempre tengan que ceder frente a otros, ocasionando, en último término, la desaparición de los derechos "perdedores". De esta forma, un conflicto entre derechos no se resuelve considerando algunos más relevantes que otros, de manera abstracta, sino mediante la ponderación de ellos en el caso concreto.

De igual modo, también resulta complejo determinar el contenido del derecho a la vida, es decir qué protege el derecho a la vida. Es así como, entre los autores, puede encontrarse distintas concepciones sobre qué es lo que protege este derecho, opiniones que es posible identificar con las teorías internas y externas de los derechos fundamentales, con los límites o contornos de los derechos. Esta toma de partido, lejos de ser inocua, genera consecuencias sumamente importantes al abordar la posibilidad la eutanasia y suicidio asistido, ello porque en algunas de sus interpretaciones, de efectuarse tales conductas, no se estaría afectado el derecho a la vida, principal argumento para prohibir la eutanasia y el suicidio. Figueroa(20) ha sistematizado las concepciones en relación al contenido del derecho a la vida. Indica que es posible encontrar diversas posturas, tales como que el derecho a la vida consiste en un derecho a vivir, a permanecer con vida; derecho a vivir bien o con dignidad; derecho a recibir lo mínimamente lo necesario para no morir inmediatamente; derecho a que no me maten y, por último, derecho a que no me maten arbitrariamente. Como puede notarse, de aceptarse que el contenido del derecho a la vida consiste en que no me maten arbitrariamente, tanto el suicidio asistido como la eutanasia no serían atentados contra el derecho a la vida, pues la voluntad y las condiciones en que pueda encontrarse una personas (enfermedad, por ejemplo) eliminaría, o al menos reduciría considerablemente, la arbitrariedad.

En último término, es preciso referirse a la posibilidad de renunciar a los derechos fundamentales, en este caso al derecho a la vida. Se trata de un tema relativamente discutido en la doctrina y sobre el cual no existe completo acuerdo. En términos generales, es posible afirmar que existe gran consenso respecto de que la titularidad de los derechos es irrenunciable(21), mientras que, en relación a la renuncia al ejercicio de los derechos, se presentan múltiples opciones y matices. De esta forma, siguiendo a Riffo(22), se puede encontrar tres modelos de renuncia a los derechos fundamentales, los cuales se diferencian principalmente en los casos y exigencias para que la renuncia al ejercicio sea válida.

Con todo, el caso del derecho a la vida es todavía más complejo, por cuanto la renuncia al ejercicio del derecho a la vida conlleva aparejada la renuncia a la titularidad de tal derecho, circunstancia que solo ocurre en este caso y no en otros. Así, 
por ejemplo, respecto de la integridad física, es posible permitir una agresión (renuncia al ejercicio del derecho), más no por ello se pierde la posibilidad futura de poder ejercerlo (renuncia a la titularidad).

De esta forma, según lo indicado anteriormente, la renunciabilidad (o irrenunciabilidad) de los derechos es considerada un obstáculo para la despenalización de la eutanasia y suicidio asistido, pero puede ser superado desde las múltiples perspectivas analizadas.

2. La titularidad y el ejercicio de los derechos de los menores de edad

Entre la doctrina, no hay duda que los menores de edad, sin importar su edad, son titulares de todos los derechos fundamentales reconocidos a las personas en Tratados Internacionales y Constituciones, aunque es posible sostener que existen importantes interrogantes en relación a su capacidad para ejercerlos.

Desde la perspectiva de los derechos fundamentales, a diferencia de lo que ocurre en otras materias, como las de naturaleza civil(23), la capacidad de los menores de edad es la regla general(24), mientras que la incapacidad debe obedecer a fuertes motivaciones. Esto debido a que, en caso de establecerse la incapacidad para ciertos colectivos de manera extensa, simplemente se estaría restando la protección de los derechos fundamentales a categorías de personas, en este caso a los menores de edad, idea que repugna la idea de dignidad humana, entre otros inconvenientes.

Con la adopción de la Convención Internacional sobre los Derechos del Niño, en 1989, se ha experimentado un cambio de paradigma en relación a que niños, niñas y adolescentes dejan de ser objetos de protección, para reconocérseles la calidad de sujetos de derechos.

Tal Convención establece como piedra angular de la materia el principio de "interés superior del niño" (25), sobre el cual no existe claridad en su contenido. Empero, es posible establecer que tal principio, derecho y norma de procedimiento ${ }^{10}$,

\footnotetext{
${ }^{10}$ Observación General No 14 (2013) del Comité de los Derechos del Niño.
}

aunque concepto indeterminado, combina dos principios que pueden resultar contrapuestos: el clásico principio de protección de los/as niños/ as y la debida autonomía de los mismos en los asuntos que les empecen(26). Este último principio puede verse reflejado, además, en el derecho a expresar su opinión libremente cuando estén en condiciones de formarse un juicio propio, consagrado en el artículo 12 de la Convención. Tal artículo debe ser entendido no solo como un derecho, sino como una guía para interpretar todos los derechos, pero sobre todo es la plasmación del deber estatal de tener en cuenta sus opiniones. De esta forma, niños, niñas y adolescentes deben participar de las decisiones que les empecen, lo que incluye la expresión de sus ideas, pero especialmente la debida consideración de las mismas ${ }^{11}$.

Por otra parte, existe coincidencia entre los autores en relación a la imposibilidad de establecer de antemano y de manera abstracta el contenido de este "interés superior"(25), en consecuencia, debe ser evaluado caso a caso en la situación concreta(27). Esta dificultad se ve aumentada debido al carácter progresivo del ejercicio por sí de los derechos fundamentales que poseen los menores de edad, el cual ha sido reconocido por la Convención pertinente(27), pues, por ejemplo, aunque reconozcamos la titularidad del derecho a la integridad física de niños, niñas y adolescentes, resulta razonable que el mismo no pueda ejercerse por sí mismo tomando la decisión de realizarse un tatuaje a los cuatro ańos, circunstancia diversa a lo que ocurre con un joven de dieciséis años.

Debido a que no poseemos criterios fijos para determinar en qué medida se va desarrollando esta progresividad de los derechos, se ha dejado en evidencia la necesidad de contar con criterios adicionales para establecerlo, empeño en el cual cobra relevancia el concepto de "madurez". El análisis de la madurez ha sido incluido en algunos textos jurídicos, tales como el Convenio de Oviedo $^{12}$, que exige ponderar su grado cuando los destinatarios son menores de edad. En otras palabras, uno de los criterios, mas no el único,

\footnotetext{
${ }^{11}$ Observación General No 12 (2009) del Comité de Derechos del Nin̄o.

${ }^{12}$ Convenio para la protección de los derechos humanos y la dignidad del ser humano con respecto a las aplicaciones de la Biología y la Medicina del Consejo de Europa (1997).
} 
para colaborar con la debida progresividad de los derechos fundamentales de niños, niñas y adolescentes es la madurez.

Existen múltiples estudios desde la psicología, la medicina y la neurociencia para medir este grado de madurez que, según se indicó, sería clave a la hora de ejercer los derechos fundamentales. Así, mientras algunos sugieren la existencia de una edad relativamente fija en la cual los adolescentes están en condiciones de tomar decisiones en materias sanitarias (15 años)(28), otros afirman que es posible determinar este grado caso a caso, sea mediante aplicación de cuestionarios o con investigaciones médicas que midan la realidad concreta(27); pero también se ha sugerido la conveniencia de establecer franjas de edad orientativa para evaluar la madurez(29) o una escala móvil de $\operatorname{edad}(30)$.

De esta forma, entender a niños, niñas y adolecentes como sujetos de derechos, implica que el ejercicio de los mismos, lo que incluye su eventual renuncia, no puede negarse a priori por su sola condición, sino que es necesario realizar un análisis acabado en el caso particular, ello debido al carácter progresivo de sus derechos. En otras palabras, no es posible descartar de modo general la posibilidad de que los menores de edad puedan solicitar válidamente la práctica de la eutanasia o suicidio asistido en casos determinados.

\section{Conclusiones}

Entre la doctrina no existe acuerdo sobre la conceptualización de la eutanasia ni sobre el suicidio asistido. De igual modo, los distintos países han regulado de diversas maneras la temática, permitiendo tal opción, en algunos casos, a los/as menores de edad.

En nuestro país, desde el año 2004 se han presentado ocho proyectos de ley sobre la materia, los que pueden dividirse entre los que permiten la eutanasia/suicidio asistido a menores de edad (3 proyectos) y los que niegan tal posibilidad (5 proyectos).

En relación a los argumentos jurídicos que frecuentemente se esgrimen para prohibir tal regulación, es posible sostener que suelen centrarse en dos aspectos, el primero de ellos hace alusión al contenido, calidad y posibilidad de renuncia del derecho a la vida, mientras que el segundo se refiere a la forma en que se ejercen (o no) los derechos fundamentales de los menores edad. Ambos temas pueden ser abordados desde la perspectiva de la teoría general de los derechos fundamentales.

Así, tradicionalmente se ha señalado que no es posible legislar permitiendo la eutanasia y el suicidio asistido debido a que nuestro sistema jurídico consagra en la Constitución Política de la República la protección del derecho a la vida. En este sentido, se ha interpretado que tal derecho posee también la calidad de deber, que protegería, en consecuencia, aun contra de la voluntad de su titular y que debido a su importancia no es posible que el ejercicio del derecho a la vida se renuncie. Sin embargo, se trata de interpretaciones que pueden ser desvirtuadas con base en los postulados de la actual teoría de los derechos fundamentales, tales como la imposibilidad de que un derecho fundamental obligue a su titular, es decir que el derecho a la vida sea al mismo tiempo un deber para su titular; la impropiedad de sostener que el mismo titular pueda vulnerar su propio derecho; la imposibilidad de sostener la existencia de jerarquía entre los derechos; la reconocida posibilidad de renunciar al ejercicio de ciertos derechos en casos puntuales, entre otros.

Por otra parte, es posible encontrar argumentos contrarios a la legalización de la eutanasia y el suicidio asistido en menores de edad basándose precisamente en esta última calidad. Frente a tal panorama, sostener, sin mas, que debe negarse tal posibilidad a los/as menores de edad sin ningún tipo de distinción, resulta contrario a la Convención Internacional sobre los Derechos del Niño, por cuanto en la misma se les reconoce como titulares de todos los derechos A mayor abundamiento, la negativa puede contravenir principio rector del tal Convención, el interés superior del niño, el derecho a ser oído y la debida participación en los asuntos que les empecen. De igual manera, si se sostiene que tales instituciones implican una renuncia al derecho a la vida, no es dable sostener a priori que los/as menores de edad carezcan de tal posibilidad; ello se debe a que la capacidad iusfundamental es la regla general y cualquier alteración, 
en este caso debido a la minoría de edad, debe ser evaluada de manera pormenorizada e interpretada restrictivamente. Pareciera ser que la solución, que ha sido estudiada y adoptada en otras latitudes, debe necesariamente considerar el concepto de "madurez" a la hora de evaluar la legalización de la eutanasia y suicidio asistido en menores de edad, puesto que tal análisis se muestra respetuoso de la progresividad de la capacidad de los niñas, niñas y adolescentes.

De esta forma, al no prohibir expresamente tal conducta la propia Carta Fundamental, se debe interpretar sus preceptos a la luz de los Tratados Internacionales y los Derechos Fundamentales en general, actividad que abre un enorme abanico de posibilidades, las cuales solo podrían desplegarse en caso de existir voluntad política de modificar la actual situación de los/as menores de edad que han solicitado la eutanasia o el suicidio asistido en nuestro país.

\section{Agradecimientos}

Fondecyt de Iniciación a la Investigación (11170788): Configuración de las Bases del Derecho Antidiscriminatorio en Chile desde sus principios jurídicos.

Proyecto de Investigación Asociativa (IAF17003): Factores asociados a mortalidad por suicidio en niños y adolescentes de América Latina: Revisión sistemática de la literatura.

\section{Referencias}

1. Beca JP, Leiva A. ¿Podría ser aceptable la eutanasia infantil? Revista Chilena de Pediatría 2014; 85(5): 608-612.

2. Misseroni A. Cuestiones jurídicas en torno al concepto de eutanasia. Acta Bioethica 2000; 8(2): 246- 263.

3. Lorda PS, Barrio Cantalejo, IM, et al. Ética y muerte digna: propuesta de consenso sobre un uso correcto de las palabras. Revista Calidad Asistencial 2008; 23(6): 271-285.

4. Ciccone L. La ética y el término de la vida humana. En Polaino Lorente A. (ed.). Manual de bioética general. Madrid: Ediciones Rialp; 1994: 423-438.

5. Grupo de Estudios de Ética Clínica de la Sociedad Médica de Santiago. Eutanasia y Acto médico. Revista Médica de Chile 2011; 139: 642-654.

6. De Miguel Sánchez C, López Romero A. Eutanasia y suicidio asistido: conceptos generales, situación legal en Europa, Oregón y Australia (I). Revista Medicina Paliativa 2006; 13(4): 207-215.

7. Singer P. Ética Práctica. 2a ed. Cambridge: Cambridge University Press; 1995.

8. Instititut Borja de Bioética Universidad Ramón Llull. Hacia una posible despenalización de la eutanasia. Declaración del Institut Borja de Bioètica. Revista Bioètica y Debat 2005; 39: 1-7.

9. OMS. Desarrollo en la adolescencia. En: Salud de la madre, el recién nacido, del niño y del adolescente. Disponible en http://www.who.int/maternal_child_adolescent/topics/adolescence/dev/es/. Fecha última consulta: 22 de enero de 2018.

10. Vivanco A. La autonomía de la persona frente al derecho a la vida no incluye el derecho a ser muerto por un tercero: La solicitud de asistencia al suicidio y el caso de Diane Pretty. Acta Bioethica 2002; 8(2): 299-313.

11. Zúniga A. Derechos del paciente y eutanasia en Chile. Revista Chilena de Derecho 2008; 21(2): 111-130.

12. Bognetti G. The concept of human dignity in European and US constitutionalism. En: Nolte G. (Ed.). European and US Constitutionalism. Cambridge: Cambridge University Press; 2005: 85-107.

13. Gómez Lugo Y. La dignidad Humana en la Jurisprudencia del Tribunal Supremo de los Estados Unidos. En: Chueca R. (Ed.). Dignidad Humana y Derecho Fundamental. Madrid: Centro de Estudios Políticos y Constitucionales; 2015: 81-121.

14. Molero M. La libertad de disponer de la propia vida desde la perspectiva constitucional. Madrid: Centro de Estudios Políticos y Constitucionales; 2014.

15. Ugarte JJ. El derecho de la vida. Santiago de Chile: Editorial Jurídica de Chile; 2006. 
Disposición del derecho a la vida de los menores de edad - Estefanía Esparza Reyes

16. Locke J. Segundo Tratado sobre el Gobierno Civil. Madrid: Tecnos; 2010.

17. Díaz Revorio F. Derechos humanos y deberes fundamentales. Sobre el concepto de deber constitucional y los deberes en la constitución española de 1978. Revista del Instituto de Ciencias Jurídicas de Puebla, México 2011; 5(28): 278-310.

18. Alexy R. Teoría de los Derechos Fundamentales. Madrid: Centro de Estudios Políticos y Constitucionales; 2008.

19. Vivanco A. Curso de Derecho Constitucional. Tomo II. Santiago de Chile: Ediciones Universidad Católica de Chile: 2006.

20. Figueroa R. Concepto de derecho a la vida. Revista Ius et Praxis, Talca 2008; 14(1): 261-300.

21. Aldunate E. Derechos Fundamentales. Santiago de Chile: Legal Publishing; 2008.

22. Riffo E. Renuncia de Derechos Fundamentales. En: Contreras P, Salgado C. (eds.). Manual sobre derechos fundamentales. Santiago de Chile: Lom Ediciones; 2017: 349-374.

23. Vázquez-Pastor L. La Construcción de la ciudadanía del menor de edad. Valencia: Tirant lo Blanch; 2009.

24. Valero A. La libertad de conciencia del menor de edad desde una perspectiva constitucional. Madrid: Centro de Estudios Políticos y Constitucionales; 2009: 53.

25. Moreno A. Intimidad y Menores. Madrid: Centro de Estudios Políticos y Constitucionales; 2017.

26. Valero A. Constitución, libertad religiosa y minoría de edad. Valencia: Universidad de Valencia; 2004.

27. Bartolomé A. Los derechos de la personalidad del menor de edad. Navarra: Thomson Reuters; 2015.

28. Steinberg L, Cauffman E, Woolard J, Graham S, Banich M. Are Adolescents Less Mature Than Adults? American Psychologist, Washington D.C. 2009; 64(7): 583-594.

29. Gil AM. El derecho a la propia imagen del menor en internet. Madrid: Dykinson; 2013.

30. Drane JF. Las múltiples caras de la competencia. En: Couceiro A. (Ed.). Bioética para clínicos. Madrid: Editorial Triacastela; 1999: 164-175.

Recibido: 27 de junio de 2018

Aceptado: 23 de agosto de 2018 